\section{Distribuição geográfica dos médicos no Brasil: uma análise a partir de um modelo de escolha locacional}

\author{
Geographic distribution of physicians in Brazil: \\ an analysis based on a locational choice model
}

Luciano Póvoa 1

Mônica Viegas Andrade 1

\author{
1 Centro de Desenvolvimento \\ e Planejamento Regional, \\ Universidade Federal \\ de Minas Gerais, \\ Belo Horizonte, Brasil. \\ Correspondência \\ L. Póvoa \\ Centro de Desenvolvimento \\ e Planejamento Regional, \\ Universidade Federal \\ de Minas Gerais. \\ Av. Francisco Sales 183, \\ apto. 602, Belo Horizonte, $M G$ \\ 30150-220, Brasil. \\ povoa@cedeplar.ufmg.br
}

\section{Abstract}

The primary aim of this study was to analyze the geographic distribution of physicians among the Regions and States of Brazil using a locational choice model. Our individual data analysis showed a positive relationship between the number of physicians per 1,000 inhabitants in a State and the number of places in residency programs. Thus, the concentration of residency programs in some States has contributed to the unequal distribution of physicians in Brazil. There are also significant differences in physicians' income between Regions.

Physicians; Physicians Distribution; Choice Behavior

\section{Introdução}

A distribuição geográfica dos médicos influencia o bem-estar social uma vez que estes são os principais provedores dos serviços de saúde. Contudo, a distribuição dos médicos resultante do processo individual de escolha locacional nem sempre coincide com a distribuição considerada socialmente adequada. O que muitas vezes se observa é que, mesmo que a oferta total de médicos em um país apresente uma relação médico/habitante adequada, a distribuição desses profissionais tende a ser concentrada em certas regiões, gerando um resultado socialmente indesejado.

O entendimento dos determinantes da distribuição geográfica dos médicos tem sido objeto de estudo em vários países 1,2,3,4,5,6. Os principais fatores destacados na literatura como sendo importantes na escolha locacional do médico são as oportunidades de mercado de trabalho disponíveis para o cônjuge, caso seja casado 7,8,9, e o local onde o médico recebeu seu treinamento, ou seja, onde fez a graduação e/ou sua residência médica 4,10,11,12,13,14.

Do ponto de vista de política pública, o entendimento dos determinantes da distribuição geográfica dos médicos é fundamental, uma vez que a evidência empírica sugere que os mecanismos de mercado são insuficientes para garantir uma distribuição geográfica ótima que atenda, ao mesmo tempo, aos parâmetros 
técnicos (redução de custos decorrentes do tamanho do estabelecimento de saúde e do número de serviços nele prestado) e às necessidades de acesso. Deste modo, a regulação da oferta de ensino médico pode gerar ganhos de bemestar social.

Os poucos estudos que tratam do problema da distribuição geográfica dos médicos no Brasil 15,16,17 limitam-se a evidenciar esta desigualdade sem, no entanto, analisar seus determinantes. Um estudo importante é o de Pinto \& Machado 18, que aponta evidências de que os médicos tendem a permanecer no local onde realizaram sua residência médica, independentemente de serem ou não naturais do local. Este resultado vem ao encontro das evidências empíricas já apontadas na literatura internacional, reforçando a importância da consideração da distribuição geográfica das faculdades de medicina e dos programas de residência médica para o entendimento da distribuição geográfica dos médicos.

O objetivo deste artigo é analisar os determinantes da decisão locacional dos médicos no Brasil, de forma a contribuir para o entendimento da sua distribuição geográfica, considerando a Unidade da Federação como unidade geográfica de escolha.

\section{Distribuição dos médicos no Brasil: evidências das desigualdades}

A Tabela 1 apresenta o número de médicos por mil habitantes, o PIB per capita (referente ao ano de 2000) e a participação de cada região e de cada Estado no total da população e no total de médicos em 2001. Ao compararmos a participação de cada região no total da população brasileira e a sua participação no total de médicos, notamos que as regiões Sudeste e Nordeste são as que possuem o maior descompasso entre estes percentuais. Enquanto o Nordeste possui $28 \%$ da população do Brasil e conta com apenas $16,2 \%$ do total de médicos, o Sudeste apresenta a maior concentração desses profissionais, possuindo aproximadamente $42 \%$ da população brasileira e quase $60 \%$ dos médicos. Esta desigualdade também se verifica entre os Estados. Os Estados de São Paulo e Rio de Janeiro concentram $46,16 \%$ dos médicos do Brasil, sendo que este último conta com $8,45 \%$ da população brasileira e possui quase $20 \%$ do total de médicos.

Uma das formas de se avaliar a disparidade entre a participação de cada Estado no total da população e no total de médicos é calcular o número de médicos por mil habitantes e com- pará-lo com o recomendado pela Organização Mundial da Saúde (OMS) - um médico para cada mil habitantes. Enquanto o Norte e o Nordeste apresentam números pouco acima do recomendado pela OMS, a Região Sudeste conta com 2,81 médicos por mil habitantes, ou seja, quase o triplo do recomendado e 2,5 vezes mais que o Norte.

Os dados da Tabela 1 indicam também uma relação positiva entre o número de médicos por mil habitantes em um estado e o seu PIB per capita. Em cada região, os Estados com o maior PIB per capita são também os que possuem os mais elevados números de médicos por mil habitantes. O Maranhão era o Estado com menor número de médicos por mil habitantes em 2001 $(0,65)$ e o que possuía o menor PIB per capita (R\$ 1.629,00), enquanto, no outro extremo estava o Distrito Federal, com 5,54 médicos por mil habitantes e um PIB per capita de R\$14.425,00.

A literatura destaca a existência de uma relação entre o número de médicos e o desenvolvimento econômico de uma região. Como apontado em Rosko \& Broyles 19, a teoria econômica sugere que a escolha locacional é influenciada por diferenças nos ganhos monetários entre as regiões. Espera-se que os médicos sejam atraídos para localidades onde os ganhos sejam mais elevados e que o aumento da quantidade de médicos em uma localidade tenda a reduzir os ganhos devido ao aumento da competição.

Os artigos de Fein 20, Rimlinger \& Steele 2 e Benham et al. 21 mostram que a taxa de médicos per capita aumenta com a renda do local e que, se ocorrer uma equalização regional da renda per capita, a desigual distribuição geográfica dos médicos será suavizada. A despeito da evidência acerca da relação entre o número de médicos per capita e o desenvolvimento econômico local, uma das implicações dos modelos de Rimlinger \& Steele é que um aumento na oferta total de médicos, não necessariamente, levará a uma melhora na sua distribuição geográfica, podendo ocorrer um aumento no número de médicos em áreas de escassez, mas o aumento será ainda maior em áreas onde já existe grande oferta. Como as localizações geográficas não são igualmente atrativas, ou seja, cada uma oferece uma combinação diferente de benefícios não pecuniários (ou amenidades), o papel dos fatores econômicos, ainda que importante, pode não ser dominante na decisão locacional.

Um aumento da oferta de médicos e o conseqüente aumento da competição no mercado de trabalho podem alterar o padrão de localização desses profissionais. Alguns estudos têm mostrado que as forças competitivas são predo- 
Distribuição dos médicos por regiões e Estados. Brasil, 2001.

\begin{tabular}{|c|c|c|c|c|}
\hline $\begin{array}{l}\text { Região/Unidade } \\
\text { da Federação }\end{array}$ & $\begin{array}{c}\text { Percentual da } \\
\text { população do Brasil }\end{array}$ & $\begin{array}{l}\text { Percentual } \\
\text { dos médicos }\end{array}$ & $\begin{array}{l}\text { Médicos por } \\
1.000 \text { habitantes }\end{array}$ & $\begin{array}{c}\text { PIB per capita } \\
(2000)\end{array}$ \\
\hline Norte & 7,70 & 4,10 & 1,12 & 3.926 \\
\hline Rondônia & 0,82 & 0,50 & 1,28 & 4.077 \\
\hline Acre & 0,33 & 0,15 & 0,92 & 3.055 \\
\hline Amazonas & 1,68 & 0,98 & 1,20 & 6.710 \\
\hline Roraima & 0,20 & 0,14 & 1,49 & 3.443 \\
\hline Pará & 3,68 & 1,90 & 1,07 & 3.054 \\
\hline Amapá & 0,29 & 0,14 & 0,99 & 4.126 \\
\hline Tocantins & 0,69 & 0,33 & 0,99 & 2.117 \\
\hline Nordeste & 28,00 & 16,20 & 1,20 & 3.019 \\
\hline Maranhão & 3,32 & 1,04 & 0,65 & 1.629 \\
\hline Piauí & 1,67 & 0,71 & 0,88 & 1.875 \\
\hline Ceará & 4,38 & 2,29 & 1,08 & 2.799 \\
\hline Rio Grande do Norte & 1,63 & 1,20 & 1,53 & 3.347 \\
\hline Paraíba & 2,01 & 1,48 & 1,53 & 2.682 \\
\hline Pernambuco & 4,65 & 3,75 & 1,67 & 3.678 \\
\hline Alagoas & 1,66 & 1,18 & 1,47 & 2.488 \\
\hline Sergipe & 1,05 & 0,58 & 1,15 & 3.318 \\
\hline Bahia & 7,67 & 3,98 & 1,08 & 3.688 \\
\hline Sudeste & 42,60 & 57,70 & 2,81 & 8.788 \\
\hline Minas Gerais & 10,52 & 9,75 & 1,92 & 5.934 \\
\hline Espírito Santo & 1,83 & 1,80 & 2,04 & 6.951 \\
\hline Rio de Janeiro & 8,45 & 19,59 & 4,82 & 9.581 \\
\hline São Paulo & 21,83 & 26,57 & 2,53 & 10.013 \\
\hline Sul & 14,80 & 14,20 & 1,99 & 7.708 \\
\hline Paraná & 5,62 & 5,24 & 1,93 & 6.898 \\
\hline Santa Catarina & 3,16 & 2,30 & 1,51 & 7.921 \\
\hline Rio Grande do Sul & 5,98 & 6,62 & 2,30 & 8.357 \\
\hline Centro-oeste & 6,90 & 7,80 & 2,34 & 6.576 \\
\hline Mato Grosso do Sul & 1,22 & 1,09 & 1,84 & 5.708 \\
\hline Mato Grosso & 1,49 & 0,88 & 1,23 & 5.362 \\
\hline Goiás & 2,97 & 2,57 & 1,80 & 4.330 \\
\hline Distrito Federal & 1,22 & 3,25 & 5,54 & 14.425 \\
\hline
\end{tabular}

Fonte: IBGE (Censo Demográfico 2000); Ministério da Saúde (DATASUS - IDB 2002).

minantes na localização dos médicos 22,23,24,25. De acordo com os autores, quando uma região alcança um certo nível de densidade de médicos, o efeito da competição torna-se dominante, fazendo com que os médicos menos qualificados tenham de se localizar em áreas menos aglomeradas e mais afastadas dos grandes centros urbanos.

\section{A distribuição geográfica dos programas de residência médica no Brasil}

O local onde o médico realizou os seus estudos influencia a sua escolha de onde morar pelo fato deste, provavelmente, já ter estabelecido no local laços sociais e profissionais importantes na carreira médica, na qual o sucesso profissional depende da formação de uma rede de pacientes. Este é um elemento passível de controle por órgãos reguladores. Países como Inglaterra e Estados Unidos controlam a abertura de 
novos cursos de medicina observando previamente a necessidade de mais médicos. No Brasil, no entanto, não existe uma regulamentação para a abertura de novas faculdades de medicina nem de cursos de residência médica que seja de acordo com as necessidades regionais.

Ao tomarmos o número de alunos no primeiro ano de residência em 2003 no Brasil como sendo uma proxy para o número de vagas nos programas de residência médica (dados da Comissão Nacional de Residência Médica), notamos uma grande concentração regional. Podemos inferir que nas regiões Sul e Sudeste estão quase $80 \%$ das vagas em programas de residência médica do Brasil. Como a residência médica é a principal modalidade de pós-graduação escolhida pelos médicos brasileiros (cerca de $75 \%$ 17) é de se esperar que médicos de outras regiões estejam migrando para o Sul e Sudeste em busca deste treinamento. Portanto, dada a tendência a permanecer no local de treinamento, a concentração das vagas de residência médica nas regiões Sul e Sudeste parece estar contribuindo para a desigual distribuição geográfica dos médicos no Brasil.

\section{Metodologia}

\section{Base de dados}

No momento da realização deste trabalho, não havia uma fonte de dados sobre médicos no Brasil com informações específicas acerca de suas características de migração. Os dados do Conselho Federal de Medicina, apesar de conterem informações sobre a localização dos médicos e sua especialização, não disponibilizam informações sobre a migração dos mesmos. Desta maneira, para estimar o modelo utilizamos os dados da PNAD (Pesquisa Nacional por Amostra de Domicílios), realizada pelo IBGE (Instituto Brasileiro de Geografia e Estatística), que fornece uma ampla variedade de informações individuais, como educação, trabalho, renda e características de migração. O período de análise é de 1997 a 2001 e foi definido pela disponibilidade de dados sobre o número de médicos e as características de cada Unidade da Federação disponibilizados pelo sistema de dados DATASUS 26 (nos anos em que ocorre a realização do censo não é feita a PNAD). As principais limitações deste banco de dados são a impossibilidade de identificar a cidade onde o médico mora (apenas a Unidade da Federação) e a sua especialidade.

Na Tabela 2 estão relacionadas as variáveis do modelo a ser estimado e a justificativa da inclusão de cada variável. Duas variáveis importantes referentes às características dos indivíduos são a idade do médico e a variável parceiro conterrâneo (que é uma variável dummy com valor igual a 1 para parceiros que nasceram na mesma Unidade da Federação e igual a zero caso contrário). A decisão de escolha locacional pode ser interpretada como uma forma de investimento e assim, quanto mais jovem é o indivíduo, mais tempo ele poderá usufruir os benefícios de uma mudança. Desta forma, indivíduos mais jovens são mais propensos a mudar. Como a variável estado civil não nos informa se os médicos já estavam casados antes da mudança para a atual Unidade da Federação de residência, ela aparece apenas como uma variável de controle.

Para tentar captar se os indivíduos já estavam unidos antes da mudança, incluímos uma variável que indica se os médicos são conterrâneos de seus parceiros. Assim, a variável parceiro conterrâneo procura captar o fato de que a decisão de mudar deve considerar também as preferências e as perspectivas profissionais do parceiro. A influência do cônjuge é apontada na literatura como sendo um fator crítico na escolha locacional dos médicos, pois a falta de oportunidades profissionais para o parceiro, especialmente aqueles que possuem níveis mais elevados de escolaridade, dificulta a escolha de pequenas comunidades e de regiões isoladas para atuar.

As rendas individuais foram divididas pelo total de horas trabalhadas gerando a variável renda por hora. Esta ponderação fez-se necessária pelo fato de que em nossa amostra os médicos do sexo masculino trabalhavam, em média, quarenta horas a mais por mês do que as do sexo feminino. Para permitir comparações ao longo do período, todos os dados monetários foram colocados a preços de setembro de 2001 (utilizamos o Índice Nacional de Preço ao Consumidor - INPC - para deflacionar as rendas individuais e o PIB per capita).

Uma variável dummy foi construída para os médicos cujo parceiro possui ocupação na área de saúde (médico, dentista, enfermeiro diplomado, acadêmico de hospital e outras atividades ligadas ao hospital não diplomadas).

Para a realização deste trabalho adotamos a premissa de que médicos generalistas e especialistas respondem de forma semelhante às alterações nas variáveis explicativas. A adoção desta premissa justifica-se pela indisponibilidade de informações sobre a formação complementar dos médicos em nossa amostra. 
Variáveis do modelo a ser estimado e a justificativa da inclusão de cada variável.

\begin{tabular}{|c|c|}
\hline Variável & Justificativa \\
\hline \multicolumn{2}{|l|}{ Características dos indivíduos } \\
\hline Sexo & Variáveis de controle \\
\hline \multicolumn{2}{|l|}{ Estado civil* } \\
\hline \multicolumn{2}{|l|}{ Trabalha para o governo* } \\
\hline $\begin{array}{l}\text { Condição no domicílio } \\
\text { (chefe, cônjuge ou filho)* }\end{array}$ & $\begin{array}{l}\text { Médicos que ainda são filhos no domicílio estão ligados às decisões } \\
\text { de localização dos pais }\end{array}$ \\
\hline $\begin{array}{l}\text { Ocupação do cônjuge na área } \\
\text { de saúde* }\end{array}$ & $\begin{array}{l}\text { Médicos com parceiros que também atuam na área da saúde tendem } \\
\text { a conseguir compatibilizar as perspectivas profissionais com a mudança }\end{array}$ \\
\hline Parceiro conterrâneo* & $\begin{array}{l}\text { Procurar captar a dificuldade em compatibilizar as vantagens da mudança } \\
\text { para ambos }\end{array}$ \\
\hline Ano* & Para verificar as mudanças no padrão de localização dos médicos no período \\
\hline Idade & A propensão a mudar difere entre indivíduos mais jovens e mais velhos \\
\hline Idade elevada ao quadrado & $\begin{array}{l}\text { Procura captar a não-linearidade entre o aumento da idade e a queda } \\
\text { da propensão a mudar }\end{array}$ \\
\hline Número de componentes na família & Quanto maior o número de componentes, maior o custo de uma mudança \\
\hline \multicolumn{2}{|l|}{$\begin{array}{l}\text { Características das Unidades } \\
\text { da Federação }\end{array}$} \\
\hline População & Proxy do tamanho da demanda por serviços médicos \\
\hline Médicos por mil habitantes & Indica a concentração de médicos \\
\hline Leitos por mil habitantes & Proxy da estrutura de trabalho disponível \\
\hline Residentes & $\begin{array}{l}\text { Proxy das possibilidades de aperfeiçoamento. (Número de alunos } \\
\text { no primeiro ano de residência médica) }\end{array}$ \\
\hline PIB per capita & $\begin{array}{l}\text { Os indivíduos procuram mudar para uma Unidade da Federação com nível } \\
\text { econômico mais elevado, pois nelas a renda esperada tende a ser maior }\end{array}$ \\
\hline
\end{tabular}

*Variável dummy.

\section{Modelo analítico}

Nocera \& Wanzenried 25 propõem que a distribuição geográfica dos médicos seja estudada como um resultado de um processo de escolha locacional no qual o indivíduo escolhe uma região que lhe proporcione o maior bemestar. Neste trabalho propomos um modelo de escolha locacional dos médicos que associa as características dos médicos às características de sua atual localidade, para estimar a probabilidade de um médico em um determinado estado ser considerado não-natural. Supondo que o médico escolhe onde se localizar procurando maximizar seu bem-estar, podemos inferir que o local onde o médico está residindo revela sua preferência locacional 24.

Por limitação do banco de dados, consideramos a Unidade da Federação, e não o município, como sendo a unidade de escolha locacional do médico. Para analisar a probabilidade do médico ser não-natural utilizamos um modelo Probit de escolha binária 27 . A variável dependente é $y_{m i}$ que assume os valores:
- $y_{m i}=1$ (não-natural) se o médico $m$ não nasceu na Unidade da Federação em que reside e está morando nela há menos de dez anos.

- $y_{m i}=0$ (natural) se o médico $m$ nasceu na Unidade da Federação em que reside ou está nela há mais de dez anos.

Como a nossa base de dados não nos permite calcular a idade que o indivíduo possuía quando mudou de Unidade da Federação caso tenha se mudado há mais de dez anos, classificamos como não-natural os médicos que não nasceram na Unidade da Federação em que residem e estão morando nela há menos de dez anos, para evitar ter em nossa amostra médicos que se mudaram ainda crianças, provavelmente pela decisão dos seus pais.

O modelo foi estimado com base no empilhamento de dados transversais que não acompanham o mesmo indivíduo ao longo do tempo (este método também é chamado de pooled cross-section, que consiste na fusão de vários bancos de dados transversais, cada um apresentando amostras aleatórias de indivíduos em momentos diferentes do tempo). Os dados arranjados desta forma podem ser tratados como 
uma base de dados transversais simples acrescentada de variáveis dummy para os anos, de forma a levar em conta as mudanças agregadas no tempo.

A estimação dos parâmetros do modelo utilizou os dois conjuntos de variáveis explicativas relacionadas na Tabela 2: (a) variáveis relacionadas às características dos médicos (vetor $\mathrm{xm}$ ); e (b) variáveis relacionadas às características das Unidades da Federação (vetor xi). A especificação do modelo é descrita da seguinte forma:

$$
\begin{aligned}
& \operatorname{Prob}\left(\mathrm{y}_{m i}=1 \mid \mathrm{x}^{\mathrm{m}}, \mathrm{x}^{\mathrm{i}}, \text { ano }\right)=\Phi\left(\beta_{0}+\beta^{\prime}{ }_{m} \mathrm{x}^{\mathrm{m}}+\right. \\
& \left.\beta_{i}^{\prime} \mathrm{x}^{\mathrm{i}}+\text { ano }\right)
\end{aligned}
$$

\section{Resultados}

\section{Descrição dos dados amostrais}

Foram identificados 1.959 médicos na amostra (PNADs dos anos 1997-1999 e 2001), dos quais $8,83 \%$ foram classificados como não-naturais (173 médicos). A Tabela 3 apresenta a composição da amostra de acordo com o sexo e a condição de naturalidade. Na amostra, 50,18\% dos médicos são do sexo masculino e estes representam 48,55\% dos não-naturais. Desta forma, existe um certo equilíbrio entre o número de médicos do sexo masculino e feminino, tanto para a amostra total quanto para os médicos não-naturais. Assim, parece não haver uma distinção entre naturalidade por sexo.

Ao analisar a média de idade na amostra, verificamos que médicos não-naturais possuem uma média de idade de 36 anos (com desvio padrão de 9,3 anos) enquanto a dos naturais é de 41 anos (desvio padrão de 10,7 anos), o que

Tabela 3

Composição da amostra por sexo.

\begin{tabular}{lccc}
\hline Sexo & Natural & Não-natural & Total \\
\hline Feminino & 887 & 89 & 976 \\
Percentual do total feminino & 90,88 & 9,12 & 100,00 \\
Percentual do total geral & 49,66 & 51,45 & 49,82 \\
& & & \\
Masculino & 899 & 84 & 983 \\
Percentual do total masculino & 91,45 & 8,55 & 100,00 \\
Percentual do total geral & 50,34 & 48,55 & 50,18 \\
Total & 1.786 & 173 & 1.959 \\
Percentual do total & 91,17 & 8,83 & 100,00 \\
\hline
\end{tabular}

Fonte: PNAD; elaboração própria. sugere que a decisão de se mudar para outra Unidade de Federação pode ser influenciada pela idade do indivíduo.

A Tabela 4 apresenta algumas características gerais dos médicos em nossa amostra. Podemos notar que o percentual de médicos "casados" é maior entre os não-naturais (65,32\%). Notamos também que há uma tendência dos médicos em ter parceiros com atividades relacionadas à área da saúde $(29,47 \%)$, sendo que esse porcentual atinge $41,59 \%$ entre os não-naturais.

Uma característica importante a ser destacada é o fato de que entre os não-naturais o porcentual de médicos que possui parceiros nascidos na mesma Unidade da Federação (conterrâneos) é significativamente inferior ao porcentual entre os naturais $(27,17 \%$ e $45,8 \%$, respectivamente). Esses dados sugerem uma influência do parceiro na decisão de mudar.

Em nossa amostra verificamos que existe uma diferença na renda real/hora média entre as regiões e também entre os médicos não-naturais e naturais. Para realizar as comparações entre as médias de renda real/hora entre nãonaturais e naturais em cada região foram feitos testes de médias.

Em todas as regiões, a renda real/hora média dos naturais é superior ou estatisticamente igual a dos não-naturais na amostra total. A renda real/hora média é mais elevada no Sul entre os naturais ( $\mathrm{R} \$ 26,80$ ) e mais elevada no Norte entre os não-naturais (R\$23,80).

Entretanto, se considerarmos apenas os médicos mais jovens (arbitrariamente selecionados aqueles com idade menor ou igual a 35 anos), notamos que a renda real/hora média se altera entre as regiões e entre os não-naturais e naturais. Neste caso, tanto no Norte quanto no Centro-oeste a renda real/hora média é mais elevada entre os não-naturais. Estas são também as regiões com o maior percentual de médicos não-naturais $(18,36 \%$ no Norte e $27,3 \%$ no Centro-oeste). O Norte permanece como a região onde os médicos não-naturais recebem, em média, a renda real/hora mais elevada $(\mathrm{R} \$ 23,30) \mathrm{e}$ o Nordeste, onde ela é menor ( $\mathrm{R} \$ 16,10)$.

Como vimos, a idade média dos não-naturais é inferior à dos naturais, o que sugere que os médicos mais jovens são mais propensos a mudar de Unidade da Federação que os mais velhos. Quando comparamos a renda real/hora média dos médicos mais jovens, notamos que em geral a média é mais elevada entre os nãonaturais, o que poderia indicar a existência de um "prêmio" para os médicos que se mudam para outra região 28 . 


\section{Resultados do modelo analítico}

A Tabela 5 apresenta os resultados do modelo, considerando os efeitos marginais da probabilidade de um médico ser não-natural (para obter variâncias robustas utilizou-se o estimador Huber/White/sandwich 27). Os coeficientes da coluna $(\mathrm{dF} / \mathrm{dx})$ nos dão os efeitos marginais, ou seja, como cada variável afeta a probabilidade de um médico ser não-natural e se a afeta positiva ou negativamente.

O sinal do coeficiente da variável idade está de acordo com o argumento de que a mudança é uma forma de investimento e indivíduos mais jovens são mais propensos a mudar, pois assim aproveitarão mais tempo o retorno do investimento. Desta forma, quanto maior a idade, menor a probabilidade de um médico ser considerado não-natural em um estado. Com o aumento da idade, a redução na probabilidade torna-se cada vez menor, conforme podemos observar pelo sinal positivo do coeficiente da variável idade elevada ao quadrado.

Os resultados do modelo também estão de acordo com as análises de Nocera \& Wanzenried 25 e Newhouse et al. 22, em que os médicos procuram evitar regiões que já alcançaram um certo nível de densidade de profissionais médicos. Nestas regiões, o efeito da competição torna-se considerável, fazendo com que os médicos procurem áreas menos aglomeradas. Assim, a probabilidade de um médico ser nãonatural é maior em estados com menos médicos por mil habitantes.

Estados que oferecem mais vagas em programas de residência atraem mais médicos em busca de aperfeiçoamento. Esta atração é captada pelo modelo por meio do sinal positivo do coeficiente da variável residentes, indicando que a probabilidade de um médico ser não-natural é maior em estados que oferecem mais vagas. Desta maneira, podemos sugerir a concentração dos programas de residência nas regiões Sul e Sudeste como sendo um dos fatores que promovem a desigual distribuição geográfica dos médicos. Este resultado sugere que políticas de atração e fixação de médicos baseadas na implantação de programas de residência médica em estados que ainda não o possuem e na expansão e aperfeiçoamento dos programas já existentes das regiões Norte e Nordeste podem ser eficazes.

O coeficiente da variável PIB per capita apresentou-se significativo em nosso modelo, indicando que os fatores econômicos exercem influência na escolha locacional dos médicos, conforme destacado anteriormente. Os resultados sugerem que quanto maior o PIB per ca-
Tabela 4

Composição da amostra: características gerais.

\begin{tabular}{lccc}
\hline & Natural (\%) & Não-natural (\%) & Total (\%) \\
\hline $\begin{array}{l}\text { Estado civil } \\
\quad \text { Solteiro }\end{array}$ & 36,23 & 34,68 & 36,09 \\
Casado & 63,77 & 65,32 & 63,91 \\
Parceiro com atividade & & & \\
na área de saúde & & & \\
Sim & 28,27 & 41,59 & 29,47 \\
Não & 71,73 & 58,41 & 70,53 \\
Conterrâneos & & & \\
Sim & & & 44,16 \\
Não & 45,8 & 27,17 & 55,84 \\
\hline
\end{tabular}

Fonte: PNAD; elaboração própria.

pita de um estado, maior a probabilidade de um médico ser não-natural. Assim, podemos associar a desigual distribuição dos médicos ao desigual desenvolvimento econômico entre as regiões e estados brasileiros.

Os resultados do modelo indicam uma influência do cônjuge na decisão locacional dos médicos, indo de encontro à ênfase dada pela literatura 3,9. Como existe uma dificuldade em conciliar as expectativas de ganhos profissionais e pessoais para ambos com uma mudança, o bem-estar do médico depende do bemestar de seu parceiro. Desta forma, a probabilidade do médico ser não-natural é inferior quando ele e seu cônjuge são conterrâneos.

Conforme sugerido pela análise descritiva dos dados, a chance de um médico possuir um parceiro que atua na área de saúde é maior entre os não-naturais (o que é confirmado pelo sinal positivo do coeficiente da variável parceiro com atividade na área de saúde). Podemos levantar duas hipóteses para este fato. Caso os parceiros já estejam juntos antes de se mudarem, fica mais fácil conciliarem as expectativas de ganhos com a mudança se os parceiros atuam em áreas afins. Outra maneira é considerar que os parceiros se conheceram depois da mudança. Por ser proveniente de outro estado, o médico estabelece contato com maior facilidade no novo estado com profissionais de áreas afins. Devemos ressaltar que estas explicações são apenas especulações, pois acreditamos que estejam envolvidos fatores sociológicos mais complexos.

Ao contrário do que se podia esperar, o tamanho populacional de um estado relaciona- 
Resultados do modelo Probit (efeitos marginais).

\begin{tabular}{|c|c|c|c|c|}
\hline Variáveis explicativas & $d F / d x$ & Desvio padrão & $\mathbf{z}$ & Prob(z) \\
\hline Idade & $-0,011$ & 0,00 & $-3,47$ & 0,001 \\
\hline Idade elevada ao quadrado & 0,0001 & 0,00 & 2,42 & 0,016 \\
\hline Número de componentes na família & $-0,006$ & 0,00 & $-1,12$ & 0,263 \\
\hline Estado civil & 0,011 & 0,02 & 0,63 & 0,526 \\
\hline Trabalha para o governo & 0,014 & 0,01 & 1,46 & 0,145 \\
\hline População & $8,89 e-09$ & 0,00 & $-4,09$ & 0,000 \\
\hline Médicos/mil habitantes & $-0,034$ & 0,01 & $-3,57$ & 0,000 \\
\hline Residentes & 0,0001 & 0,00 & 3,45 & 0,001 \\
\hline Conterrâneos & $-0,061$ & 0,01 & $-5,38$ & 0,000 \\
\hline Parceiro com atividade na área de saúde & 0,032 & 0,02 & 2,33 & 0,020 \\
\hline Chefe & 0,022 & 0,02 & 0,89 & 0,371 \\
\hline Cônjuge & 0,048 & 0,04 & 1,44 & 0,150 \\
\hline Filho & $-0,070$ & 0,01 & $-3,85$ & 0,000 \\
\hline PIB per capita & $9,08 e-06$ & 0,00 & 3,37 & 0,001 \\
\hline Leitos/mil habitantes & 0,003 & 0,01 & 0,40 & 0,692 \\
\hline Sexo & 0,015 & 0,01 & 1,19 & 0,234 \\
\hline d97 & $-0,011$ & 0,02 & $-0,68$ & 0,496 \\
\hline d98 & $-0,017$ & 0,01 & $-1,18$ & 0,238 \\
\hline d99 & $-0,020$ & 0,01 & $-1,24$ & 0,214 \\
\hline Pseudo R2 $=0,1894$ & & & & \\
\hline
\end{tabular}

se negativamente com a probabilidade de um médico ser não-natural. Este resultado pode estar refletindo as transformações ocorridas no Brasil nas ultimas décadas, relacionadas à construção de Brasília e à criação de estados como Mato Grosso do Sul e Tocantins que, apesar de possuírem populações relativamente pequenas, exerceram atração sobre vários indivíduos na expectativa de ganhos decorrentes da criação de novas oportunidades.

Entre as variáveis que apresentam características dos indivíduos, o número de componentes da família não é significativo a pelo menos $90 \%$ de confiança, o mesmo acontecendo com as dummies para sexo, estado civil, trabalha para o governo e as dummies para ano. A variável número de leitos por mil habitantes em cada estado também não apresentou um coeficiente significativo.

\section{Considerações finais}

Este artigo procurou descrever e analisar a distribuição geográfica e a decisão locacional dos médicos no Brasil. Os resultados indicam que os médicos mais jovens são mais propensos a mudar e que a estrutura de ensino médico de um estado, mais especificamente, a oferta de vagas em programas de residência médica, atraem médicos em busca de aperfeiçoamento. Desta maneira, podemos indicar a concentração dos programas de residência nas regiões Sul e Sudeste como sendo um dos fatores que promovem a desigual distribuição geográfica dos médicos.

A probabilidade de um médico ser não-natural é mais elevada em estados com maior PIB per capita, o que sugere uma atração exercida pelo fator econômico. O papel do cônjuge também se mostrou significativo na decisão locacional do médico, confirmando o destaque dado pela literatura.

Por fim, os resultados apontam uma significativa diferença de atração exercida pelas regiões do Brasil. O Norte o e Centro-oeste destacam-se como regiões onde a probabilidade de um médico ser não-natural é maior, e o Nordeste mostrou-se como um mercado pouco atrativo para os migrantes. 


\section{Resumo}

A localização dos médicos exerce influência no bemestar de uma comunidade, pois, estes são provedores diretos dos serviços de saúde. Contudo, a distribuição dos médicos resultante do processo de escolha locacional realizado pelos mesmos, muitas vezes não coincide com o que seria considerado socialmente adequado. O objetivo deste artigo é, por meio de um modelo Probit de escolha binária, modelar a decisão locacional dos médicos com base nos dados da PNAD e analisar o papel de alguns fatores destacados na literatura como sendo importantes para a compreensão de sua escolha locacional e, conseqüentemente, da sua distribuição geográfica no Brasil. Os resultados apontam uma atração exercida pelas vagas em programas de residência, indicando que a probabilidade de um médico ser não-natural é maior em Estados que oferecem mais vagas. Desta maneira, podemos indicar a concentração dos programas de residência nas regiões Sul e Sudeste como sendo um dos fatores que promovem a desigual distribuição geográfica dos médicos. O Norte e o Centro-oeste destacam-se como regiões atrativas para médicos migrantes.

Médicos; Distribuição de Médicos; Comportamento de Escolha

\section{Colaboradores}

L. Póvoa contribuiu na coleta, análise dos dados e na redação do artigo. M. V. Andrade orientou a pesquisa, contribuiu na análise dos dados e na redação do artigo.

\section{Agradecimentos}

Agradecemos o apoio da Coordenação de Aperfeiçoamento de Pessoal de Nível Superior por meio da bolsa de Mestrado.

\section{Referências}

1. Pitblado JR, Pong RW. Geographic distribution of physicians in Canada. Ottawa: Health Canada; 1999.

2. Rimlinger GV, Steele HB. An economic interpretation of the spatial distribution of the physicians in the US. South Econ J 1963; 30:1-12.

3. Kristiansen IS, Forde OH. Medical specialists' choice of location: the role of geographical attachment in Norway. Soc Sci Med 1992; 34:57-62.

4. Nigenda G. The regional distribution of doctors in Mexico, 1930-1990: a policy assessment. Health Policy 1997; 39:107-22.

5. Goic A. Distribución geográfica de los médicos en Chile. Rev Med Chil 1995; 123:306-11.

6. Kobayashi Y, Takaki H. Geographic distribution of physicians in Japan. Lancet 1992; 340:1391-3.

7. Holmes JE, Miller DA. Factors affecting decisions on practice locations. J Med Educ 1986; 61:721-6.

8. Leonardson G, Lapierre R, Hollingsworth D. Factors predictive of physician location. J Med Educ 1985; 60:37-43.

9. Kazanjian A, Pagliccia N. Key factors in physicians' choice of practice location: findings from a survey of practitioners and their spouses. Health Place 1996; 2:27-34.
10. Cooper JK, Heald K, Samuels M. The decision for rural practice. J Med Educ 1972; 47:939-44.

11. Cooper JK, Heald K, Samuels M. Affecting the supply of rural physicians. Am J Public Health 1977; 67:756-9.

12. Scheffler RM. The relationship between medical education and the statewide per capita distribution of physicians. J Med Educ 1971; 46:955-98.

13. Burfield WB, Hough DE, Marder WD. Location of medical education and choice of location of practice. J Med Educ 1986; 61:545-54.

14. Watson CJ. The relationship between physician practice location and medical school area: an empirical model. Soc Sci Med 1980; 14D:63-9.

15. Rigatto M. O que falta ao Rio Grande do Sul em matéria de médicos: quantidade, qualidade ou distribuição geográfica? Rev Assoc Méd Bras 1966; 12:505-12.

16. Melo OC. Aspectos quantitativos e qualitativos da formação de médicos e plano de redistribuição geográfica dos mesmos. Rev Assoc Méd Bras 1971; 17:323-30.

17. Machado MH, coordenador. Perfil dos médicos no Brasil: relatório final. Rio de Janeiro: Fundação Oswaldo Cruz/Conselho Federal de Medicina/ 
Ministério da Saúde/Programa das Nações Unidas para o Desenvolvimento; 1996.

18. Pinto LF, Machado MH. Médicos migrantes e a formação profissional: um retrato brasileiro. Rev Bras Educ Méd 2000; 24:53-64.

19. Rosko MD, Broyles RW. The economics of health care: a reference handbook. New York/London: Greenwood; 1988.

20. Fein R. Studies on physician supply and distribution. Am J Public Health 1954; 44:615-24.

21. Benham L, Maurizi A, Reder MW. Migration, location and remuneration of medical personnel: physicians and dentists. Rev Econ Stat 1968; 50: $332-47$.

22. Newhouse JP, Williams AP, Bernett BW, Schwartz WB. Does the geographical distribution of physicians reflect market failure? Bell Journal of Economics 1982; 13:493-506.

23. Schwartz WB, Newhouse JP, Bernett BW, Williams AP. The changing geographic distribution of boardcertified physicians: Facts, theory and implications. Santa Monica: The Rand Corporation; 1980.
24. Williams AP, Schwartz WB, Newhouse JP, Bernett BW. How many miles to the doctor? N Engl J Med 1983; 309:958-63.

25. Nocera S, Wanzenried G. On the dynamics of physician density: theory and empirical evidence for Switzerland. Bern: University of Bern; 2002.

26. Instituto Brasileiro de Geografia e Estatística. Pesquisa de assistência médico-sanitária (AMS), Rio de Janeiro, 1999 e 2002. Rio de Janeiro: Instituto Brasileiro de Geografia e Estatística; 2002.

27. Greene WH. Econometric analysis. $4^{\text {th }}$ Ed. Upper Saddle River: Prentice Hall; 2000.

28. Póvoa LMC. Dois ensaios econômicos sobre a distribuição geográfica dos médicos no Brasil [Dissertação de Mestrado]. Belo Horizonte: Centro de Desenvolvimento e Planejamento Regional, Universidade Federal de Minas Gerais; 2004.

Recebido em 13/Abr/2005

Versão final reapresentada em 01/Dez/2005

Aprovado em 14/Dez/2005 